\title{
Kindliche Leukämien und EMF-Expositionen in der Umgebung hochfrequenter Sendestationen (KiSS) - Fragestellung und Studienbeschreibung
}

\author{
H. Brüggemeyer ${ }^{1}$, J. Philipp ${ }^{2}$, H. Merzenich ${ }^{3}$, S. Schmiedel ${ }^{3}$, M. Blettner ${ }^{3}$, and J. Schüz ${ }^{4}$ \\ ${ }^{1}$ Niedersächsischer Landesbetrieb für Wasserwirtschaft, Küsten- und Naturschutz, Hannover, Deutschland \\ ${ }^{2}$ Südwestrundfunk, Stuttgart, Deutschland \\ ${ }^{3}$ Institut für Medizinische Biometrie, Epidemiologie und Informatik, Universität Mainz, Deutschland \\ ${ }^{4}$ Institute of Cancer Epidemiology, Danish Cancer Society, Copenhagen, Denmark
}

\begin{abstract}
Zusammenfassung. Im Rahmen einer Fall-Kontroll-Studie wird untersucht, ob es einen Zusammenhang zwischen dem Leukämierisiko bei Kindern und der Exposition gegenüber elektromagnetischen Feldern in der Umgebung leistungsstarker Sendestationen von Rundfunk und Fernsehen gibt. Bislang wurden fünf Studien im Umkreis von Rundfunksendeanlagen durchgeführt. Deren Aussagekraft ist jedoch durch eine grobe Expositionsabschätzung und ihren ökologischen Studienansatz ohne individuelle Expositionsbestimmung limitiert. Wesentliches Element der hier vorgestellten Studie ist dagegen eine individuelle Expositionsabschätzung, die zum einen auf der Geocodierung von Wohnadressen und Expositionspunktquellen und zum andern auf den tatsächlichen Strahlungsleistungen und Betriebsdaten der Senderbetreiber beruht.
\end{abstract}

Das Studiengebiet besteht aus den Umgebungen von 17 leistungsstarken AM-Mittelwellensendern und 8 UKW-FMbzw. analogen TV-Sendern in Westdeutschland. Zur Studienpopulation gehören alle im Diagnosezeitraum von 1984 bis 2003 aufgetretenen Fälle, die im Studiengebiet leben bzw. gelebt haben. Als Fälle werden alle Kinder definiert, die zum Zeitpunkt der Diagnose zwischen 0-14 Jahren alt waren, bei denen eine primäre Leukämie diagnostiziert wurde und die dem Deutschen Kinderkrebsregister bekannt sind. Jedem Fallkind werden 3 gleich alte Kontrollkinder gleichen Geschlechts aus der gleichen Senderumgebung gegenübergestellt. Möglich wurde diese Untersuchung durch die Förderung durch Mittel des Deutschen Mobilfunkforschungsprogramms sowie durch die Mitarbeit aller relevanten Senderbetreiber und des Deutschen Kinderkrebsregisters an der Universität Mainz. Sie soll bis August 2007 abgeschlossen sein.

Correspondence to: $\mathrm{H}$. Brüggemeyer

(hauke.brueggemeyer@nlwkn-h.niedersachsen.de)

\section{Einleitung}

Mögliche gesundheitsschädigende Effekte durch nichtionisierende elektromagnetische Felder werden seit Jahrzehnten in Wissenschaft und Öffentlichkeit kontrovers diskutiert. Bislang wurden fünf Studien im Umkreis von Rundfunksendeanlagen durchgeführt: auf Hawaii (Mascarinec, 1994), in Australien (Hocking, 1996), Großbritannien (Dolk, 1997a, b), Italien (Michelozzi, 2002) und Korea (Park, 2004). Dabei ergaben sich teilweise schwache Hinweise auf ein möglicherweise häufigeres Auftreten von Leukämieerkrankungen bei Kindern im Nahbereich der Sender.

Trotzdem bieten diese Studien keine schlüssige Evidenz für einen Zusammenhang zwischen Exposition und Krebshäufigkeit. Methodische Grenzen sind die zum Teil geringen Fallzahlen sowie die teilweise zweifelhafte Vollzähligkeit der Registrierung von Krebserkrankungen. Die wesentliche Limitierung der o.g. Studien ist die grobe Expositionsabschätzung. Keine der bisherigen Studien führte eine individuelle Expositionsschätzung durch, sondern charakterisierte die Exposition für kleinräumige $\mathrm{Ge}$ biete, z.B. Stadteile, wobei dann für alle Häuser innerhalb dieses Gebietes die gleiche Exposition angenommen wurde. Diese Charakterisierung basiert zudem lediglich auf der Entfernung zur Expositionsquelle. Insbesondere bei UKW- und TV- Sendeanlagen trifft diese Annahme einer mit wachsender Entfernung monoton abnehmenden Exposition im Allgemeinen nicht zu. Die Antennen von VHF- und UHFSendern besitzen in der Regel eine ausgeprägte vertikale Bündelung Richtung Horizont und sind gewöhnlich in großer Höhe montiert. Das führt dazu, dass die expositionsrelevante Feldstärke in Bodennähe von mittleren Werten am Fuß des Sendeturms zunächst etwas ansteigt, nach wenigen

Published by Copernicus Publications on behalf of the URSI Landesausschuss in der Bundesrepublik Deutschland e.V. 
Kilometern ein Maximum erreicht und dann erst monoton abfällt. Bei Lang- und Mittelwellensendern herrschen erst in einer Distanz von einigen Wellenlängen zur Antenne einfache Fernfeldbedingungen mit einem weitgehend monotonen Abfall der elektrischen und magnetischen Feldstärke. Die Topographie hat ebenfalls einen oft nicht zu vernachlässigenden Einfluss auf die Exposition.

Noch begrenzender für die Aussagekraft der zitierten Studien ist der ökologische Studienansatz, bei dem keine individuellen Daten zugrunde gelegt werden. Vielmehr beruhen diese Studien auf der Distanz eines kleinräumigen Gebietes zur Feldquelle, wobei für alle Häuser dieses Gebietes die gleiche Expositionsstärke angenommen wird.

Die Daten des Deutschen Kinderkrebsregisters zeigen, dass in Deutschland jährlich etwa 1800 Kinder vor ihrem 15. Geburtstag an Krebs (einschließlich nicht-maligner Hirntumoren) erkranken. Bei einer jährlichen Bevölkerung von 13 Millionen Kindern dieser Altersgruppe beträgt die jährliche Inzidenz somit 14,2/100 000 Kinder. Die kumulative Inzidenz liegt bei $212 / 100000$, das heißt, etwa jedes 470. neugeborene Kind erkrankt. Häufigste Erkrankungsgruppen sind die Leukämien mit 33,8\% und einer Inzidenz von 4,7/100 000. 82,5\% der Leukämien sind akute lymphatische (ALL) und 15,1\% sind akute myeloische Leukämien (Kaatsch, 2004).

Mit den als vollständig anzusehenden Daten des Deutschen Kinderkrebsregisters und den weitgehend vollständig vorhandenen historischen Emissionsdaten von einer Vielzahl deutscher Rundfunksender bietet sich eine gute Grundlage um eine Fall-Kontroll-Studie mit individueller Expositionsermittlung durchzuführen. Die Berechung der Exposition wird in einer weiteren Veröffentlichung (Philipp, 2007) detaillierter beschrieben.

\section{Epidemiologische Untersuchungsverfahren}

Es gibt eine Reihe von epidemiologischen Studientypen mit unterschiedlichen Vor- und Nachteilen. Diese werden im Folgenden beschrieben und in Bezug auf die hier vorliegende Fragestellung bewertet.

\section{3 Ökologische Studie}

Beim ökologischen Ansatz wird untersucht, ob die Exposition verschiedener Bevölkerungsgruppen mit der Häufigkeit des Auftretens einer bestimmten Erkrankung korreliert. Es liegen dabei keine Expositionsdaten auf individueller Basis vor. Beispielsweise wird untersucht, ob in zwei Regionen, die sich aufgrund des Vorliegens einer bestimmten Exposition unterscheiden, verschiedene Erkrankungshäufigkeiten beobachtet werden. Als Erkrankungshäufigkeit wird hierzu gewöhnlich die Neuerkrankungsrate (Inzidenz) oder die Sterblichkeit (Mortalität) herangezogen.

\subsection{Vor- und Nachteile}

Der ökologische Ansatz ist i.a. mit relativ geringem Aufwand und vor allem ohne Kontakt zu betroffenen Personen möglich. Weil keine individuellen Expositionsdaten vorliegen, ist allerdings fraglich, ob die Fälle in der als exponiert betrachteten Region auch wirklich exponiert waren. Auch die Berücksichtigung möglicher Einflussfaktoren (Confounder) ist in der Regel nicht möglich. Für die Durchführung solcher Studien ist die Kenntnis der Alters- und Geschlechterverteilung der zugrunde liegenden Bevölkerung notwendig. Diese Daten liegen in Deutschland in der Regel nur auf der Basis der Gemeinden vor. Für viele Expositionsquellen ist eine Untersuchung auf Gemeindeebene aber zu grob. Des Weiteren werden in Deutschland Krebsneuerkrankungen bei Erwachsenen nicht flächendeckend erfasst. Auch die Daten der Mortalität sind auf Grund der häufiger fraglichen Diagnose auf den Leichenschauscheinen nur bedingt aussagefähig.

\section{Cluster-Studien}

Ökologische Untersuchungen auf kleinräumigen Gebieten, in denen eine Häufung vermutet wird, werden auch als Cluster-Studien bezeichnet. Dabei muss zuerst untersucht werden ob eine signifikante Häufung von Fällen vorliegt und dann ob sich diese auf eine gemeinsame Ursache zurückführen lassen könnte. Eine ,signifikante“ Häufung von Erkrankungsfällen kann allerdings auch im Rahmen von Zufallsschwankungen auftreten. Wissenschaftlich werden die aus Clusteruntersuchungen erhalten Ergebnisse als eher wenig aussagekräftig eingeschätzt, wobei sie aber zur Hypothesengenerierung für nachfolgende analytische Studien eingesetzt werden können.

\section{Fall-Kontroll-Studie}

Die Probanden von Fall-Kontroll-Studien bestehen aus einer Gruppe von Personen, die eine bestimmte Erkrankung haben, sowie einer geeigneten Kontrollgruppe von Personen, die von dieser Krankheit nicht betroffen sind. Die Exposition in beiden Gruppen wird verglichen. Der Zusammenhang zwischen einer Exposition und der Erkrankung wird dabei durch die Berechnung der Odds Ratio bestimmt. Die Odds Ratio ist das Verhältnis der Chancen der Exposition bei den Fällen zu den Chancen der Exposition bei den Kontrollen, was gleichbedeutend ist mit dem Verhältnis der Erkrankungschancen von Exponierten und Nicht-Exponierten. Eine statistisch signifikant von 1 verschiedene OR bedeutet das Vorliegen einer Assoziation zwischen Exposition und Erkrankung. 


\subsection{Vor- und Nachteile}

In der Krebsepidemiologie werden Fall-Kontroll-Studien sehr häufig durchgeführt. Gründe dafür sind vor allem, dass dieser Studientyp aufgrund der retrospektiven Betrachtungsweise eine verhältnismäßig kurze Laufzeit hat und dass man bei seltenen Erkrankungen direkt von den Erkrankungsfällen ausgehen kann.

Probleme von Fall-Kontroll-Studien sind vor allem deren potentielle Fehlerquellen: Selektionsbias, Recall bias und Confounding. Diese Störfaktoren lassen sich in der Regel bei sorgfältiger Studienplanung in der Auswertung berücksichtigen, aber nicht immer ausschließen.

\section{Kohorten-Studie}

Beim Kohorten-Ansatz, der sowohl retrospektiv als auch prospektiv sein kann, verläuft die Blickrichtung immer von der Exposition zur Erkrankung. Ein retrospektiver KohortenAnsatz ist dann möglich, wenn individuelle Expositionsdaten aus der Vergangenheit vorliegen. Bei Kohortenstudien kann das relative Risiko direkt aus der Erkrankungshäufigkeit der Kohorte in Relation zur Vergleichsbevölkerung oder aus dem Verhältnis der Erkrankungshäufigkeiten der unterschiedlichen Expositionsgruppen der Kohorte selbst berechnet werden.

\subsection{Vor- und Nachteile}

Ein wesentlicher Nachteil einer prospektiven Kohortenstudie ist der Aufwand. Zur Identifizierung eines Risikos für seltene Erkrankungen müssen sehr viele Personen, von denen nur wenige erkranken, über einen langen Zeitraum beobachtet werden. Ein besonderer Vorteil ist, dass der Verlauf von Exposition und Erkrankung mitverfolgt werden kann und so die Gefahr von Verzerrungen kleiner als in den anderen Studientypen. Aber auch hier müssen die Auswirkungen von Confoundern berücksichtigt werden.

\section{Fragestellung}

Die Hauptfragestellungen der KiSS-Studie sind:

- Gibt es einen Zusammenhang zwischen dem Leukämierisiko bei Kindern und der Exposition gegenüber elektromagnetischen Feldern in der Umgebung leistungsstarker Sendestationen von Radio und Fernsehen?

- Gibt es unterschiedliche Risiken bei verschiedenen Sendertypen in Abhängigkeit von spezifischen Expositionscharakteristiken?

\section{Studiendesign}

Bei der Entwicklung eines adäquaten epidemiologischen Studiendesigns musste berücksichtigt werden, dass

- Kinderleukämie mit einer jährlichen Inzidenz von 4,8/100 000 Kindern eine seltene Erkrankung ist,

- zur Identifizierung eines möglichen Risikopotentials durch hochfrequente Felder eine entsprechende Fallzahl gegeben sein muss (ausreichende statistische Power)

- dass individuelle Expositionsdaten vorliegen, die über eine reine Abstandsberechnung hinausgehen,

- der Anteil der Mobilfunkexpositionen an der Gesamtexposition gering sein sollte und somit zurückliegende Zeiträume erfasst werden müssen (retrospektive Expositionserfassung).

Ein Fall-Kontroll-Design mit retrospektiver Expositionserfassung ist das für die Beantwortung der Fragestellungen effizienteste Verfahren. Die Rekrutierung der Fälle erfolgt über das Deutsche Kinderkrebsregister, das seit 1984 die interessierenden Leukämieerkrankungen vollzählig erfasst (Kaatsch, 2004). Kontrollkinder werden über die Einwohnermeldeämter aus der historischen Wohnbevölkerung gezogen. Dabei wird ein Fälle-Kontrollen-Verhältnis von 1:6 angestrebt, um bei der Auswertung ein Verhältnis von 1:3 sicher erreichen zu können.

Als Studienzeitraum wurde die Zeit zwischen 1984 und 2003 angesetzt. In diesem Zeitraum muss beim erkrankten Kind erstmalig die Diagnose gestellt worden sein. Unter Berücksichtigung einer Latenzzeit zwischen Exposition und Erkrankung wird der zu berücksichtigende Expositionszeitraum als die Zeit von 1982 bis 2003 festgelegt.

Damit liegt die Hälfte des Zeitraums vor der flächendeckenden Einführung des Mobilfunks. Der digitale Rundfunk spielt während des gesamten Zeitraums als relevante Expositionsquelle keine Rolle.

Die ostdeutschen Gebiete wurden von der Untersuchung ausgeschlossen, da hierfür keine verlässliche Ziehung von Kontrollen aus der historischen Wohnbevölkerung möglich ist und auch historische Betriebsdaten ostdeutscher Sendeanlagen nur eingeschränkt zur Verfügung stehen.

Um den Aufwand bei der Ermittlung der historischen Emissionsdaten zu reduzieren wurden zuerst die Sender in den bevölkerungsschwachen Regionen ausgeschlossen. Nach der Verfügbarkeit der Daten der Sender und der Bevölkerungsverteilung wurden 17 AM-Sender und 8 FM/TV-Sender als Zentren der Studienregionen ausgewählt. Zur Definition des Studiengebietes wurde unter Zugrundelegung der Betriebsdaten für jeden AM-Sender der ungefähre $1 \mathrm{~V} / \mathrm{m}$-Radius ( $120 \mathrm{~dB}(\mu \mathrm{V} / \mathrm{m})$-Radius) bzw. der ungefähre $90 \mathrm{~dB}(\mu \mathrm{V} / \mathrm{m})$-Radius für UKW-FM- und analoge TV-Sender bestimmt. Für diese Senderregionen wurden die zugehörigen Gemeinden ermittelt, deren Gemeindegebiet mindestens zum Teil in diesem Bereich liegt (siehe Abb. 1). 


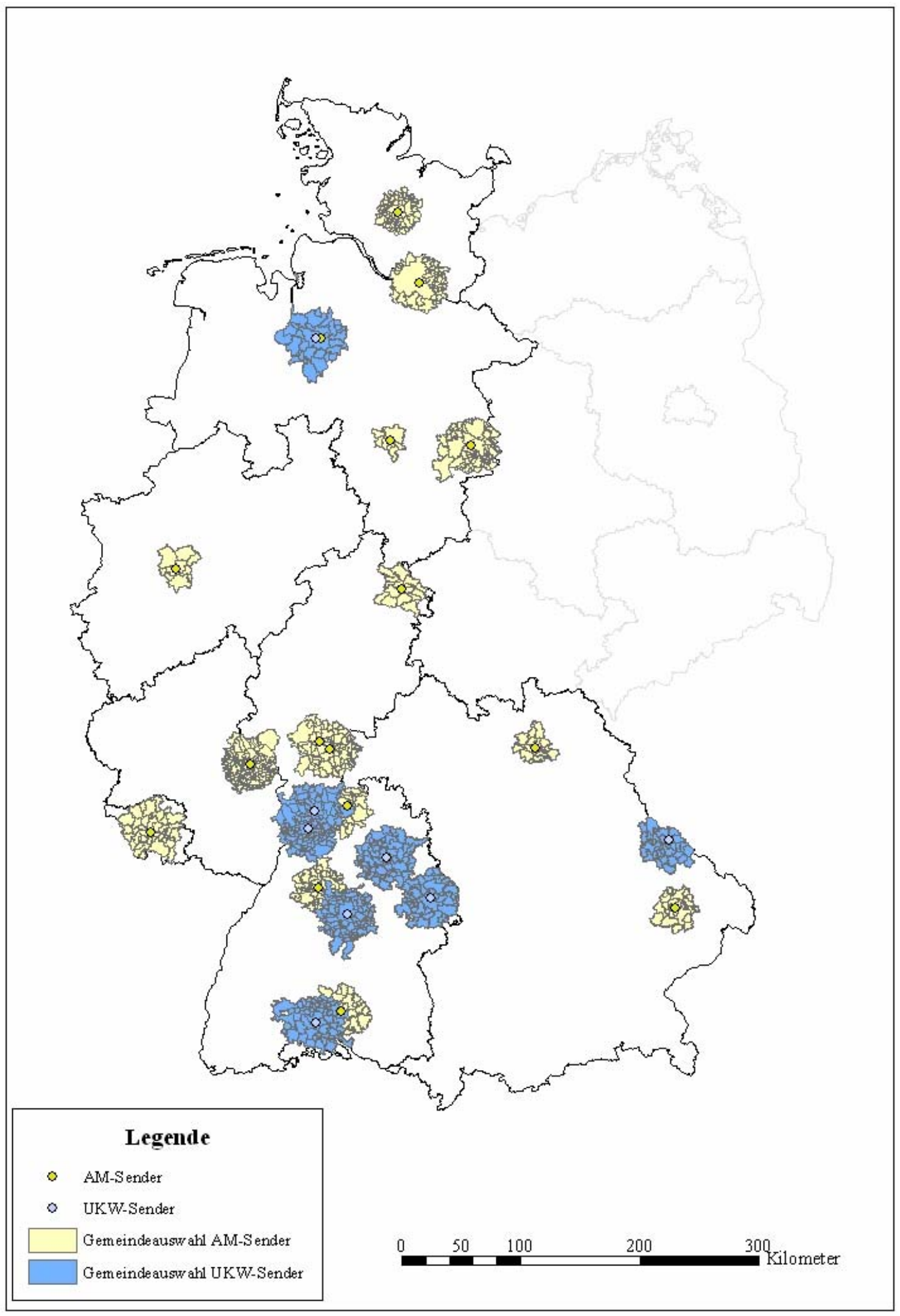

Abb. 1. Übersicht zu Standorten der AM-Sender und der UKW/TV-Sender. 


\section{Studienbeschreibung}

\section{Zur Auswahl der Fälle und Kontrollen}

\begin{tabular}{lcrr} 
Gemäß der Internationalen & \multicolumn{2}{c}{ Klassifikation der } \\
Krebserkrankungen & bei & Kindern & (ICCC-3) \\
werden & folgende & Klassifikationen & betrachtet:
\end{tabular}

ICCC 11 Lymphoid leukaemias

ICCC 12 Acute myeloid leukaemias

ICCC 13 Chronic myeloproliferative diseases

ICCC 14 Myelodysplastic syndrome and other myeloproliferative diseases

ICCC 15 Unspecified and other specified leukaemias

Die Kriterien für die Berücksichtigung eines Fallkindes in der Fall-Kontroll-Studie sind:

- Kinder, die an einer primären Leukämie (ICD C91-C95) gemäß ICCC Gruppe I (ICCC 11-15) erkrankt sind, sofern dies ihre erste Krebserkrankung darstellt (Ersttumoren bzw. primäre Leukämie);

- die bei der Diagnosestellung zwischen 0 und 14 Jahren Jahre alt waren;

- deren Krebserkrankung im Zeitraum vom 1.1.1984 bis 31.12.2003 diagnostiziert wurde;

- die dem Deutschen Kinderkrebsregister (DKKR) bekannt sind;

- die mit ihren Familien zum Zeitpunkt der Diagnosestellung ihren Wohnsitz im definierten Studiengebiet hatten.

Die Kinder, die als Kontrollen dienen, werden nach folgenden Match-Kriterien ausgewählt:

- gleiches Alter zum Zeitpunkt der Diagnose des Fallkindes;

- Geschlecht;

- Senderregion;

- Meldezeitraum in der Senderregion

Folgende Kinder sind als Kontrollkinder ausgeschlossen:

- die dem DKKR als krebskranke Kinder bekannt sind.

Mit den genannten Kriterien wurden 2015 Fallkinder ermittelt. Die entsprechende Ziehung der Kontrollen durch die zuständigen Einwohnermeldeämter ergab 12090 Kontrollkinder. Für alle Fälle und Kontrollen werden die Geokoordinaten der Wohnanschrift ermittelt, die zur individuellen Expositionsbestimmung verwendet wird.

Eine Befragung der Teilnehmer ist nicht vorgesehen. Als mögliche Störgrößen können Alter, Geschlecht, Region und Urbanität (Stadt-Land) berücksichtigt werden.
Es ist geplant, neben einer Auswertung für die ermittelte Gesamtexposition auch eine Auswertung für die Exposition getrennt nach Modulationsarten AM, FM, ATV (entsprechend den Frequenzbereichen LW/MW, UKW, Band III/IV/V) durchzuführen.

\section{Stand}

Die Ziehung der Kontrollen ist weitgehend abgeschlossen. Von der Mehrzahl der zu betrachtenden Sender liegen die relevanten Betriebsdaten vor. Diese Daten werden zurzeit auf Plausibilität geprüft; gleichzeitig werden die zur Ermittlung der Feldstärke benutzten Rechenmodelle anhand vorliegender historischer und aktueller Messungen validiert.

\section{Ausblick}

Nach Abschluss dieser Verifikation werden für jedes Teilgebiet des Studiengebietes für den gesamten Studienzeitraum flächenhaft monatliche Expositionswerte für alle relevanten Sender getrennt berechnet. Daraus werden dann für jede Geokoordinate (Fälle, Kontrollen, Verifikationspunkte) die Summenexposition für AM, FM und TV und die Gesamtexposition berechnet. Diese Ermittlung wird blind durchgeführt; dem Team, das die Exposition berechnet, ist nicht bekannt, welche der Geokoordinaten zu welcher Gruppe gehören. Der Abschluss der Studie ist für das dritte Quartal 2007 vorgesehen.

\section{Literatur}

Dolk, H., Shaddick, G., Walls, P., Grundy, C., Thakrar, B., Kleinschmidt, I., and Elliott, P.: Cancer Incidence Near Radio and Television Transmitters in Great Britain, I. Sutton Coldfield Transmitter, Am. J. Epidemiol., 145, 1-9, 1997a.

Dolk, H., Elliott, P., Shaddick, G., Walls, P., and Thakrar, B.: Cancer incidence near radio and television transmitters in Great Britain II. All high power transmitters, Am. J. Epidemiol., 145, 10-17, 1997b.

Hocking, B., Gordon, J. R., Grain, H. L., and Hatfield, G. E.: Cancer incidence and mortality and proximity to TV towers, Med. J. Aust., 165, 601-605, 1996.

Kaatsch, P. and Spix, C.: Jahresbericht 2003 des Deutschen Kinderkrebsregisters, Universität Mainz, 2004.

Maskarinec, G., Cooper, J., and Swygert, L.: Investigation of increased incidence in childhood leukemia near radio towers in Hawaii, J. Environ. Pathol. Toxicol. Oncol., 13, 33-37, 1994.

Michelozzi, P., Capon, A., Kirchmayer, U., Forastiere, F., Biggeri, A., Barca, A., and Perucci, C. A.: Adult and childhood leukemia near a high-power radio station in Rome, Am. J. Epidemiol., 155, 1096-1103, 2002.

Park, S. K., Ha, M., and Im, H. J.: Ecological study on residences in the vicinity of AM radio broadcasting towers and cancer death: preliminary observation in Korea, Int. Arch. Occup. Environ. Health, 77, 387-394, 2004. 
Philipp, J., Merzenich, H., Brüggemeyer, H., Schmidel, S., Blettner, M., und Schüz, J.: Retrospektive Bestimmung der elektromagnetischen Exposition durch analoge Rundfunksender im Rahmen von KiSS, Adv. Radio Sci., 5, 2007. 